\title{
Divertor power and particle fluxes between and during type-I ELMs in ASDEX Upgrade
}

\author{
A. Kallenbach, R. Dux, T. Eich, R. Fischer, L. Giannone, J. Harhausen, A. Herrmann, \\ H.W. Müller, G. Pautasso, M. Wischmeier, and the ASDEX Upgrade Team \\ Max-Planck-Institut für Plasmaphysik, EURATOM Association, Boltzmannstr. 2, D- \\ 85748 Garching, Germany
}

\begin{abstract}
Particle, electric charge and power fluxes for type-I ELMy H-modes are measured in the divertor of the ASDEX Upgrade tokamak by triple Langmuir probes, shunts, IR-thermography and spectroscopy. The discharges are in the medium to high density range, resulting in predominantly convective ELMs with moderate fractional stored energy losses of $2 \%$ or below. Time resolved data over ELM cycles are obtained by coherent averaging of typically one hundred similar ELMs, spatial profiles from the flush-mounted Langmuir probes are obtained by strike point sweeps. Application of simple physics models is used to compare different diagnostics and to make consistency checks, e.g. the standard sheath model applied to the Langmuir probes yields power fluxes which are compared to the thermographic measurements. In between ELMs, Langmuir probe and thermography power loads appear consistent in the outer divertor, taking into account additional load due to radiation and charge exchange neutrals measured by thermography. The inner divertor is completely detached and no significant power flow by charged particles is measured. During ELMs, quite similar power flux profiles are found in the outer divertor by thermography and probes, albeit larger uncertainties in Langmuir probe evaluation during ELMs have to be taken into account. In the inner divertor, ELM power fluxes from thermography are a factor 10 larger than those derived from probes using the standard sheath model. This deviation is too large to be caused by deficiencies of probe analysis. The total ELM energy deposition from IR is about a factor 2 higher in the inner compared to the outer divertor. Spectroscopic measurements suggest a quite moderate contribution of radiation to the target power load. Shunt measurements reveal a significant positive charge flow into the inner target during ELMs. The net number of elementary charges correlates well with the total core particle loss obtained from highly resolved density profiles. As a consequence, the discrepancy between probe and IR measurements is attributed to the ion power channel via a high mean impact energy of the ions at the inner target. The dominant contributing mechanism is proposed to be the directed loss of ions from the pedestal region into the inner divertor.
\end{abstract}

PACS numbers: 52.25.Fi, 52.55.Rk
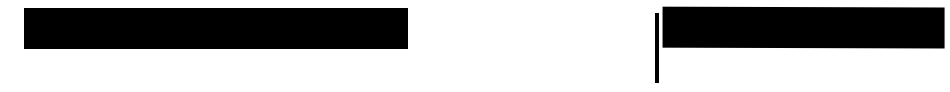


\section{Introduction}

Despite the fact that the ELMy H-mode is the standard scenario foreseen for ITER, thorough divertor characterisation and modeling over a complete ELM cycle are hardly found in the literature. One reason is the considerable experimental effort, requiring strike point sweeps and the repetition of the discharge for different diagnostic settings [1]. Since the interpretation of various diagnostics is sometimes limited (e.g., due to insufficient electron collisionality and inclination angle for Langmuir probe (LP) evaluation, or neglect of surface effects for thermography [2]), multiple measurements are required to allow for consistency checks. On the modelling side, deviations from the fluid ansatz caused by collisionless electrons or ions have been named as the possible cause for the failure to obtain a good match between code calculations and experimental data for low collisionality conditions [3].

An example of an important and unresolved problem is the in-out asymmetry of the divertor power load during ELMs. Although understanding of the edge localized mode (ELM) phenomenon has advanced considerably over the last years [4], the divertor power load asymmetry during ELMs has been an unexplained observation in many tokamaks [5], [6], [7]. For standard field direction (ion grad-B drift towards the active divertor), a larger fraction of the ELM energy loss, measured by infrared (IR) thermography, is deposited in the inner divertor compared to the outer divertor. This was in particular a surprising observation, since the instabilities associated with the ELM are predominantly active around the low-field side midplane, which has a shorter connection length to the outer divertor. In addition, in-between ELMs the outer divertor is hotter than the inner divertor and it receives generally higher power fluxes. Consequently, a mechanism has to be identified which leads to a preferential power direction into the inner divertor for standard field orientations.

Ion dynamics is proposed to be primarily responsible for the behaviour of the ELM power deposition in the divertor. Measurements of the duration of the ELM energy pulse showed good agreement with the delay time of pedestal ions to reach the divertor target [8], [9]. Pedestal ions carry in principle enough energy to explain the observed power load in the inner divertor when the collisionality is not too high. The first comprehensive modelling of the ion in-out deposition characteristics was presented by Fundamenski and coworkers [10] to describe time-averaged power load profiles in JET. The three-dimensional guiding-centre Monte-Carlo code ASCOT was used to investigate the dependence of the power load in the inner and outer divertor on collisionality, ion $\mathrm{Bx} \nabla \mathrm{B}$ drift direction and the radial electric field, $\mathrm{E}_{r}$. Indeed, conditions could be identified with a preferential power loss towards the inner divertor. Later, JET experiments with reversed current and field showed that direct ion orbit losses do not dominate the inter-ELM transport [11], but the ELM energy loss remained an outstanding issue. In a more general calculation, Hahn and coworkers suggested the large negative radial electric field as an important ingredient for directed ELM ion losses towards the inner divertor [12].

Two-dimensional simulations of the dynamic response of the scrape-off layer plasma to an ELM (neglecting impurities and impurity radiation) showed large changes of the divertor parameters related to plasma currents and ExB motions [13]. Manipulation of the target electric currents has been suggested to affect the power load due to ELMs [14]. Very recently, external MHD mode amplification due to coupling with SOL currents has been proposed as a new ELM mechanism [15] [16].

The role of the plasma sheath in energy transmission to the target was emphasised in [4], but the expected strong sheath potential rise was not accompanied by a pronounced increase of $\mathrm{T}_{e}$ during ELMs in the SOL as observed by Thomson scattering in DIII-D. Floating potentials in excess of $100 \mathrm{~V}$ have been measured during ELMs in TCV [17], in combination with target current densities approaching the ion saturation current density.

Recently, in ASDEX Upgrade (AUG) a close correlation was found between the ELM energy in-out divertor asymmetry and electric currents flowing along the SOL and through the target, suggesting a causal relationship [18]. All data shown 
in this paper are obtained in lower single-null configuration with the ion $\mathrm{Bx} \nabla \mathrm{B}$ drift directing towards the active divertor (favourable drift direction for L-H transition). Under these conditions, the inner divertor plasma is usually colder compared to the outer divertor. As a consequence, a thermoelectric current [19] [20] flows from the plasma into the inner target, through the structure and into the outer divertor plasma. The current is maintained by a superior number of (fast) electrons reaching the target in the hotter divertor and a corresponding higher ion flux towards the target in the colder divertor. The electric circuit is closed by the conducting divertor structure and current flow along SOL field lines. When the direction of $\mathrm{B}_{t}$ is switched, the original hotter outer divertor becomes the colder one and the inner divertor the hotter one. Consequently, the electric current into the target tiles changes direction. The switch of divertor temperature is not fully symmetric with $\mathrm{B}_{t}$ reversal probably due to effects of the preferential heat outflow at the low field side and different connection lengths towards the divertor targets. The thermoelectric current along the SOL field lines flows for all combinations of $\mathrm{I}_{p}$ and $\mathrm{B}_{t}$ orientations in the direction of the plasma current and bootstrap current [21]. During ELMs, strong spikes of the measured electric current are observed which are also oriented in direction of the plasma current. The combination of the AUG divertor diagnostics (IR thermography, Langmuir probes, spectroscopy in the visible range and the electric current measurements through tiles) allows the energy transport in electron and ion channels to be separated for the first time.

The data presented in this paper have been obtained in the 2006 experimental campaign of ASDEX Upgrade, with tungsten-coated plasma facing components (PFCs) and carbon strike point zones in the lower divertor. The discharges are in the medium to high density range at typically $70 \%$ of the Greenwald density, resulting in predominantly convective ELMs [22]. All discharges used deuterium as working gas.

The paper is organised as follows. After introduction of a typical ELM cycle, profiles of various plasma parameters along the outer divertor are shown for the inter-ELM phases in section 3. Corresponding measurements during ELMs for both inner and outer divertor are presented in section 4. In section 5., a quantitative analysis for electron and ion transport channels is done using a simple plasma sheath model. Various mechanisms are discussed which contribute to the divertor ELM power in-out asymmetry. Conclusions are drawn in section 6.

\section{Temporal evolution during the ELM cycle}

To introduce the time domain around a typical ELM in AUG, figure 1 shows the temporal evolution of ion saturation currents and electron temperatures in the inner and outer divertor SOL during the ELM cycle of an ELMy H-mode discharge at about $70 \%$ of the Greenwald density. The $\mathrm{H}_{98 y 2}$-factor (thermal stored energy divided by the prediction of the IPB98(y,2) scaling) being close to 1.0 and the rise of the ELM frequency at the NBI power step indicates the ELMs to be type-I. Information about pedestal diagnostics and the MHD mode structure before and during ELMs can be found in [23]. The data of figure 1 are coherent time averages with respect to start times of 166 ELMs determined by the fast rise of the thermography power load. The inner divertor exhibits much lower electron temperatures throughout the whole ELM cycle. The very low ion saturation current in-between the ELMs indicates almost complete detachment of the inner divertor. The outer divertor shows two different states after the ELM, first with low $\mathrm{T}_{e}$ and high values of $\mathrm{j}_{\text {sat }}$, later the divertor plasma adapts a hotter phase with weaker recycling, which remains until the next ELM.

\section{Profiles during inter-ELM phases}

Figure 2 shows plasma parameters in the outer divertor from flush-mounted Langmuir probes [24] and IR thermography [5] for two different after-ELM conditions of the discharge introduced in figure 1 . The spatial coordinate ds sep $_{\text {denotes the }}$

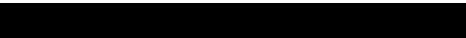



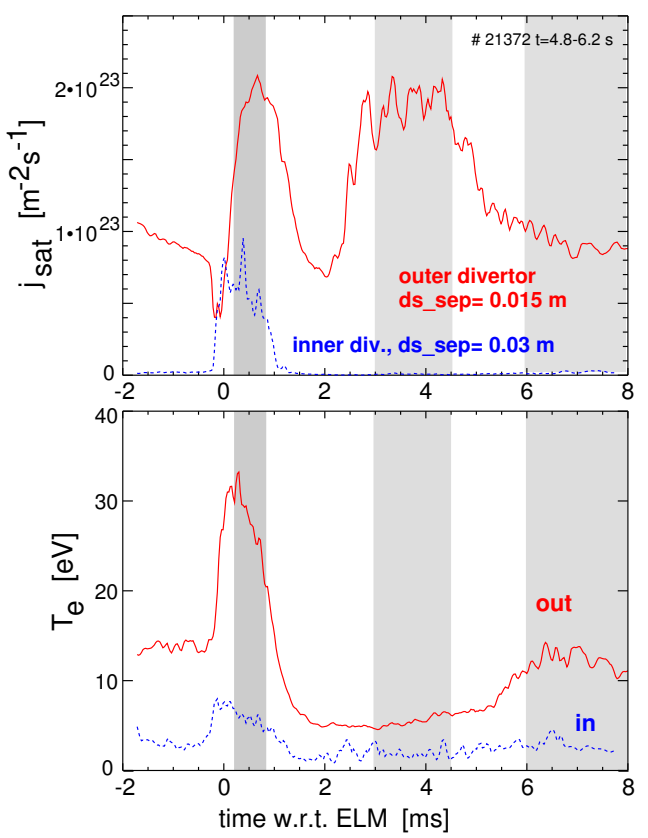

Figure 1. Ion saturation currents (top) and electron temperatures (bottom) in the outer and inner divertor SOL during the ELM cycle of type-I ELMy H-mode discharge 21372, $\mathrm{P}_{\text {heat }}=10 \mathrm{MW}, \mathrm{P}_{\text {rad }}=4 \mathrm{MW}, \mathrm{f}_{\text {Green }}=0.7, \mathrm{q}_{95}=3.3, \mathrm{I}_{p}=1 \mathrm{MA}$. The shaded time intervals are used below for profile characterisation. Locations of the measurements in the SOL are indicated by the distance to the strike point along the target, $\mathrm{ds}_{\text {sep }}$.

distance towards the strike point, measured along the target. Positive values of $\mathrm{ds}_{\text {sep }}$ refer to the scrape-off layer (SOL), negative to the private flux region (PFR).

Shortly after each ELM, a phase with cold divertor conditions develops which lasts a few ms [25]. This behaviour is typical in the present AUG divertor configuration, the cold phase is prolonged with higher gas puffing. The two phases correspond to two different recycling levels. The cold phase exhibits much stronger recycling, but the moderate electron temperatures of $5 \mathrm{eV}$ and above suggest no strong influence of recombination. The heatflux from the probes is calculated here using the standard formula [26]

$q=\left(8 k T_{e}+E_{r e c}\right) j_{s a t}$,

where $\mathrm{E}_{\text {rec }}$ is the recombination energy at the target. The measured ion saturation curent, $\mathrm{j}_{\text {sat }}$, is assumed to be equal to the ion flux density, $\Gamma_{i}$, in the following. Despite the quite large variation in $\mathrm{T}_{e}$, quite similar power fluxes are obtained from LP and IR during the different inter ELM divertor states. In the outer parts of the PFR and SOL profiles, the thermography sees more power flux, which may be explained by power deposition via radiation and CX neutrals [27].

In the inner divertor, very small power flow is measured by the Langmuir probes during the inter-ELM phases, as indicated by the vanishing ion saturation current shown in figure 1. Pressures at the inner strike point are typically two orders of magnitude below the outer midplane pressure, indicating complete detachment. The large $\mathrm{D}_{\alpha}$ photon emission usually observed in the inner divertor is attributed to recombination, in line with $\mathrm{T}_{e}$ of $1-3 \mathrm{eV}$ measured by the probes.

\subsection{Power profile widths}

The power width in the divertor is an interesting parameter from the engineering point of view as well as for comparison with values inferred from midplane electron temperature profiles. From the $\mathrm{T}_{e}^{5 / 2}$ dependence of the classical parallel 

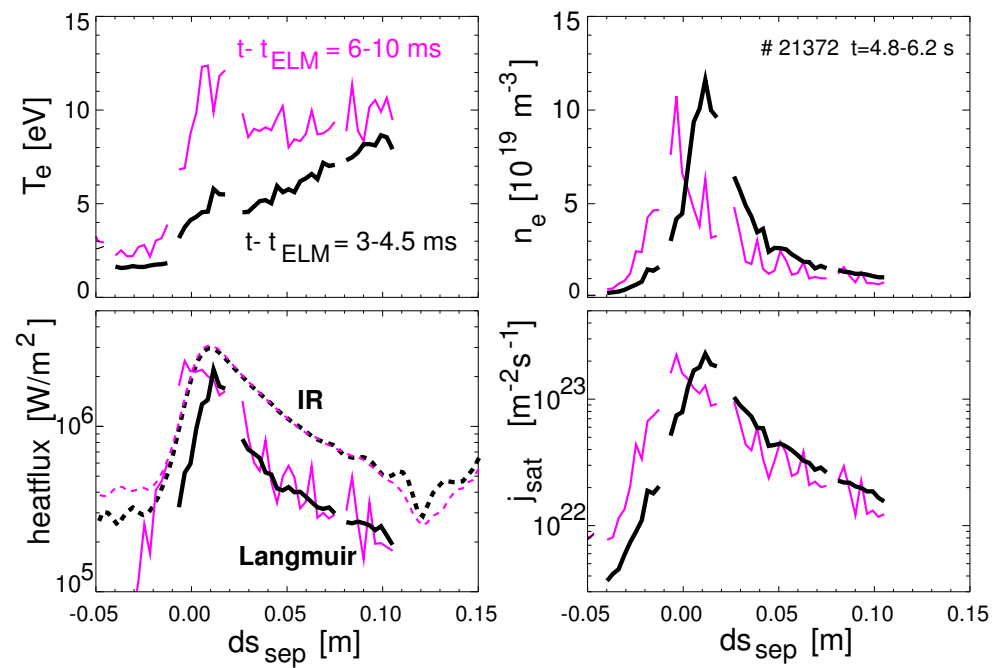

Figure 2. Profiles along the outer target of electron temperature, density, ion saturation current and heatflux from Langmuir probes and thermography. Shown are the 'hot' and the 'cold' phase in between ELMs as indicated in figure 1. Spatial coordinate ds sep $_{\text {denotes the }}$ distance from the strike point measured along the divertor target, with positive values in the SOL and negative values in the private flux region.

electron heat conductivity, the power width $\lambda_{p}=2 / 7 \lambda_{T e}$ is expected in a multi-1d model (radial sequence of flux tubes connecting midplane and target) without perpendicular transport [28]. Figure 3 shows heat flux profiles in the outer divertor for a medium and a high density discharge, evaluated from Langmuir probes and IR thermography using coherent ELM averaging as described before. To eliminate the geometrical effect on the heat flux densities along the target, the parallel heatflux is obtained by dividing the perpendicular heat flux densities by the sine of the field line impact angle. The spatial coordinate has been mapped to the outer midplane (omp). The (electron) power width obtained from the Langmuir probes according to Eq. 1 is a factor of 2 less than the one obtained from thermography. Boths widths increase with density, in line with the behaviour reported for the very similar Div II configuration in ASDEX Upgrade [29]. The power widths observed in this study (see figure 3) are in the medium to upper range of those reported in [29], which is explained by the medium to high density range in the present study.

This increase of the power width with density is also in line with the density dependence of the electron temperature decay length typically obtained from Thomson scattering measurements [28]. Midplane $\mathrm{T}_{e}$ e-folding lengths for the discharges of figure 3 obtained from edge Thomson scattering, albeit with reduced accuracy compared to measurements of ref. [28], are $6 \pm 2 \mathrm{~mm}$ for both discharges. While the power widths obtained from the probes are a factor 2-4 larger than the simple expectation $2 / 7 \lambda_{T e}$, the thermographic widths are clearly larger. The power widths measured by thermography are comparable to the poloidal ion gyroradius at the outer midplane separatrix. Assuming an ion temperature of $\mathrm{T}_{i, \text { sep }}=$ $200 \mathrm{eV}$, an ion poloidal gyroradius of $7 \mathrm{~mm}$ is obtained. Fundamenski and coworkers compared power deposition profiles in JET with various theoretical models [30], identifying the radial transport to be dominated by classical ion conduction. For ASDEX Upgrade, a considerable impact of turbulent (electron) transport on the power width is expected. A quite robust, fixed ratio of the electron density and temperature gradient lengths, $\eta_{e}=2$, is observed around the separatrix in the H-mode [31], which still awaits theoretical reproduction. 

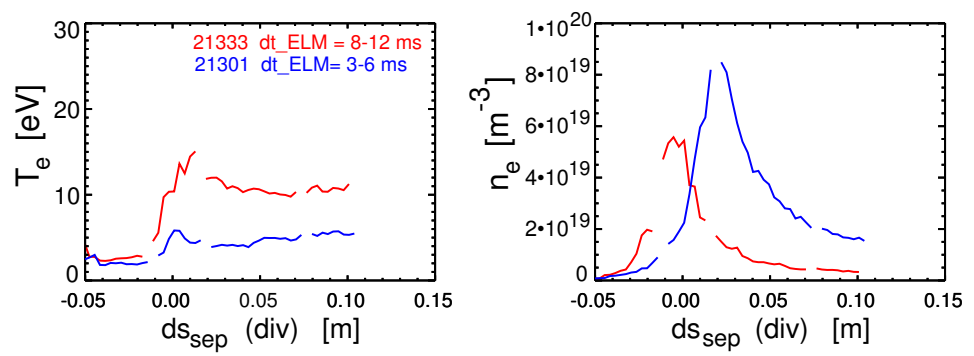

|| power fluxes, mapping to omp
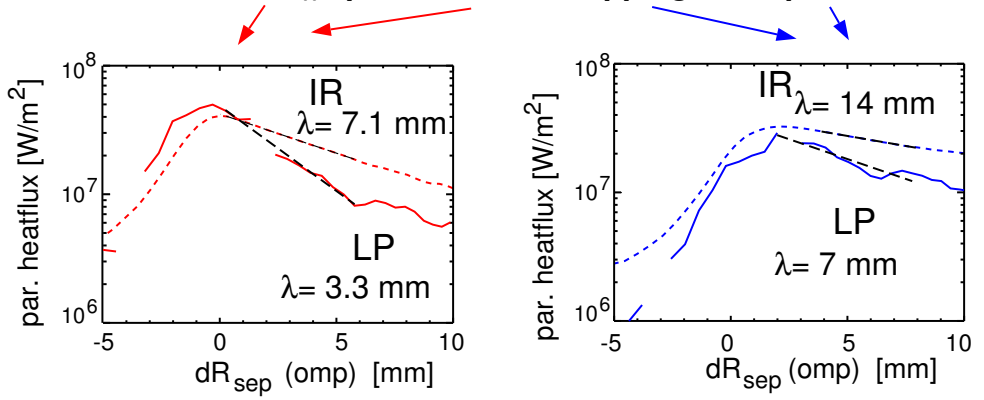

Figure 3. Top: Inter-ELM electron temperature and density profiles along the outer target for a high density (blue) and a medium density (red) H-mode discharge. Bottom: Parallel heatflux at the outer target mapped to the outer midplane (omp) from IR thermography (dashed line) and Langmuir probes (solid line) for the medium density (left) and the high density (right) discharge. The straight dashed lines indicate the decay length fits. Spatial coordinate $\mathrm{dR}_{\text {sep }}$ is the distance to the separatrix in the outer midplane at the vertical position of the magnetic axis. Data are taken during stationary phases prior to an ELM.

\section{Profiles and temporal evolution during ELMs}

In the following, the measurements described above are applied to ELMs. Since the time resolution of many diagnostics involved is not fast enough to resolve details during the ELM, profiles will be shown that are averages of data within the interval 0.2-0.8 ms after the ELM start, as defined by fast rise in the IR signal. This choice is a compromise between the wish to obtain typical ELM plasma parameters not too far away from the peak values and to cover a duration which yields integral values close to (in fact $\approx 2 / 3$ ) total ELM values. It has to be noted that the Langmuir probe data are impeded by validity limits of the evaluation model during ELMs, but this is expected to lead primarily to higher uncertainties compared to the inter-ELM cases, and not to completely misleading results. Probe data points where the measurements are not compatible to the underlying ansatz for the probe characteristics are omitted. The electron temperatures derived from the probes during the ELM are still moderate and do not exceed the limit set by the probe biasing voltage. Therefore, e.g., a dominating influence of collisionless, fast electrons is excluded. Nevertheless, applicability of the triple probe evaluation routines during an ELM has to be regarded as practical working hypothesis until a quantitative description of the nonlinear ELM phase is available.

Figure 4 shows divertor profiles along the outer and inner target during a coherently averaged ELM. Most striking is the large discrepancy between the inner divertor power load obtained from IR versus LP. The ELM deposition peaks farther away from the separatrix in the inner divertor and extends far into the SOL region. To eliminate the geometrical effect on the heat flux densities shown in figure 4, figure 5 shows a comparison of the parallel heatflux in both divertors. The profiles along both targets are plotted versus the distance of the corresponding field lines from the separatrix in the outer midplane, $\mathrm{dR}_{\text {sep }}$. The peak value of the power flux at the inner target corresponds to a field line about $5 \mathrm{~mm}$ outside the separatrix in the outer midplane. The large discrepancy between LP and thermography values suggests an increased power 

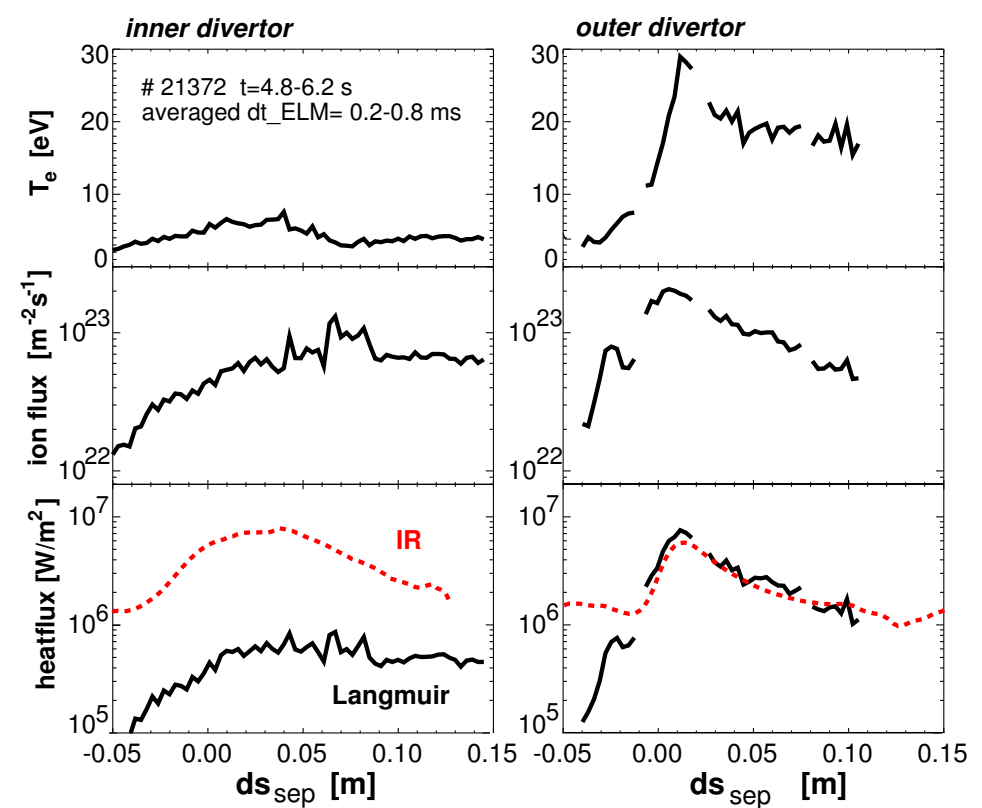

Figure 4. ELM electron temperature, ion saturation current and heatflux from standard Langmuir probe evaluation and thermography for the discharge of figure 1. Profiles are obtained via coherent ELM averaging and have been time averaged from $t=0.2-0.8 \mathrm{~ms}$ (first grey bar in figure 1) with respect to the ELM start time. Biasing voltages of the triple probes are 36 and $72 \mathrm{~V}$ for the inner and outer divertor, respectively. The evaluation of the thermography measurements includes a correction for the spatial shifts caused by a small amplitude diagnostic mirror vibration. This correction leads to somewhat lower values for the power deposition in the inner divertor compared to a previous evaluation presented in [32].

flow in the ion channel or the effect of radiation.

At this point, a possible deficiency of the thermography measurements should be adressed. Power flux measurements with IR thermography had been questioned in the past due to the possible effect of soft layers or surface topology, whose emission characteristics have the potential to falsify the derived power load [2]. Such effects are not expected to play an important role for the data presented here. First, the effect of layers is included in the evaluation software of the AUG IR thermography system [5]. In particular, the derived ELM energy is supposed to be a robust quantity, while the temporal development of the power flux may be stronger affected. Artefacts hinting towards improper treatment of surface effects, like negative power fluxes right after an ELM, are not observed in the data. Second, the AUG divertor material in the strike point zone is fine grain graphite, which is known to less affect thermography measurements compared to CFC [33].

The power e-folding lengths obtained during an ELM are just slightly increased compared to the inter-ELM phase, in accordance with earlier observations [29] and the assumptions in the ITER Physics Base [34]. For the conditions of figure 4, the IR power decay length mapped to the midplane is $7.8 \mathrm{~mm}$ during the ELM. The decay lengths obtained from the Langmuir probe tend to approach the IR value during the ELM, and is $6.2 \mathrm{~mm}$ in this case. The inter-ELM values are very similar to those shown in figure 3 . 


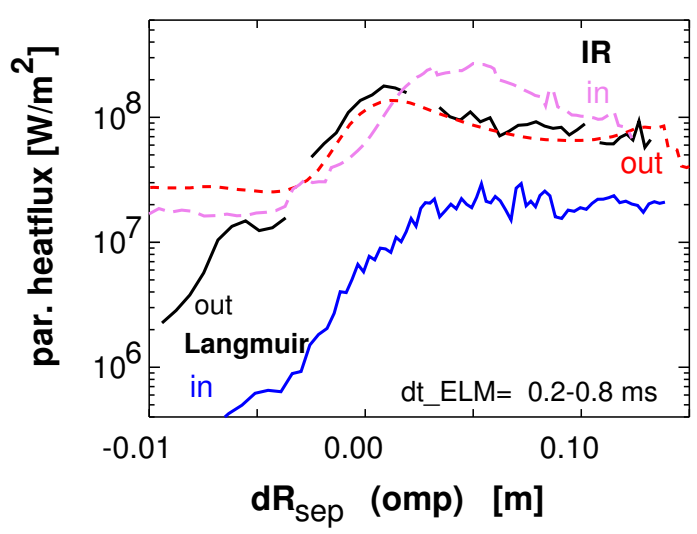

Figure 5. ELM parallel heatflux (averaged from $\mathrm{dt}_{E L M}=0.2-0.8 \mathrm{~ms}$ ) in inner and outer divertor mapped to the outer midplane (omp) corresponding to the conditions of figure 4 . The inter-ELM out/in asymmetry switches during the ELM in the thermography data only.

\subsection{Radiative power load}

Since bolometry is too slow to resolve ELMs temporally, the divertor radiation is analysed using spectroscopic measurements of $\mathrm{D}_{\alpha}$ and the CIII line at $465 \mathrm{~nm}$. Figure 6 shows the arrangement of the viewing lines in the divertor. Each line of sight measures CIII and $\mathrm{D}_{\alpha}$ emission through an arrangement consisting of a beam splitter and band pass interference filters. The total line-integrated radiation is extrapolated from the individual spectral lines using atomic data to all known

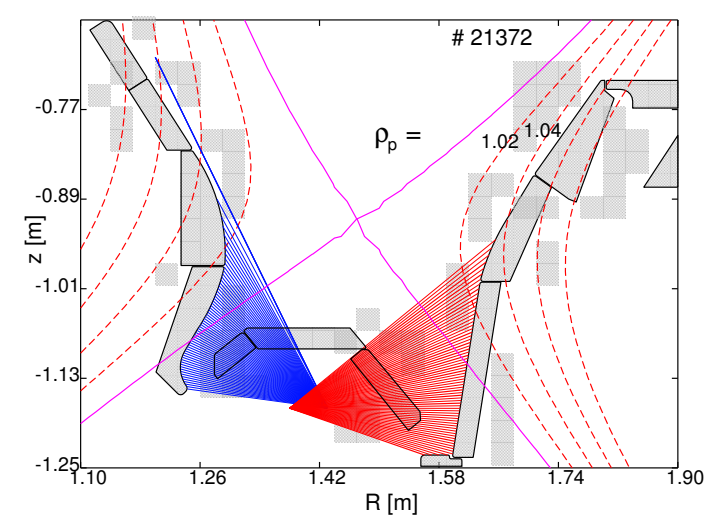

Figure 6. Arrangement of divertor spectroscopy ( $\mathrm{D}_{\alpha}$ and CIII) and equilibrium for \# 21372. In both divertors, 60 lines of sight span a region of about $0.25 \mathrm{~m}$ along the targets.

important transitions of the corresponding ionisation state [35]. The particle influx is determined from the measured spectral line using the S/XB technique [36]. Subsequently, using the inverse of S/XB, the emitted power on all other known lines is calculated using the known influx and adding up. Figure 7 shows the extrapolated line integrated power flux from neutral deuterium and carbon ions. The total power includes also an estimate for the contribution of CII obtained under the assumption of the same influx of $\mathrm{C}^{+}$as $\mathrm{C}^{2+}$. Langmuir probe measurements of $\mathrm{T}_{e}$ and $\mathrm{n}_{e}$ have been used to evaluate the atomic data S/XB. Since the electron density derived by the probes is associated with the sheath entrance where a sonic Mach=1 flow is assumed, the value has been multiplied by the factor 2 to better approximate the plasma density at the position of line emission/ionisation, which is located predominantly outside the pre-sheath region.

The inverse S/XB method works as long as recombination can be neglected, i.e. for hot, ionising divertor conditions. In addition, the ionisation time to the next higher state must be shorter than the residence time in the divertor plasma. For application to ELMs, the ionisation time must also be shorter than the ELM duration. These conditions are fulfilled for the 

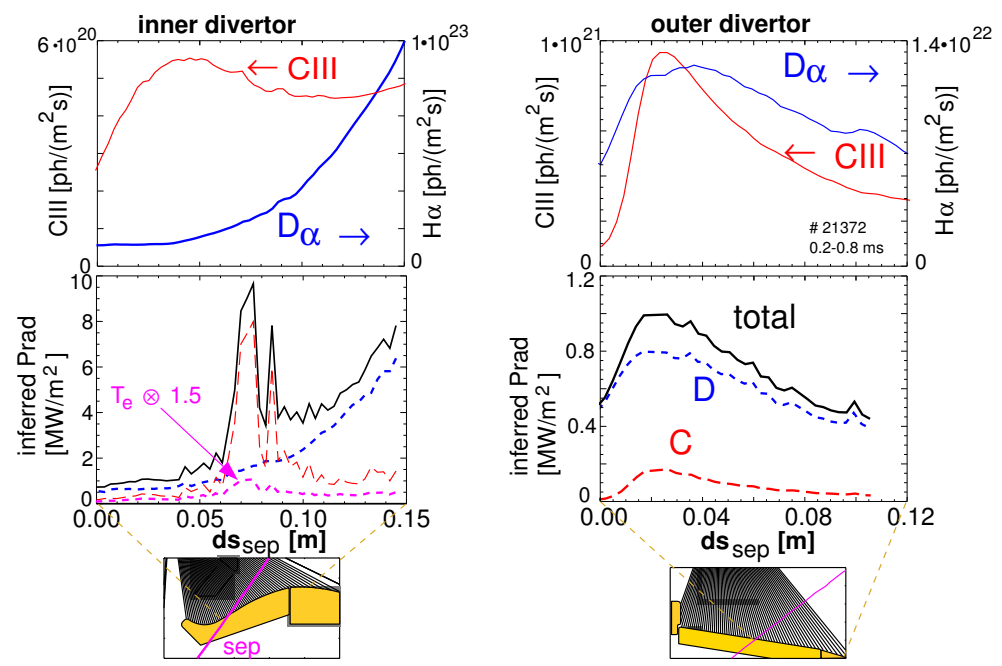

Figure 7. CIII and $\mathrm{D}_{\alpha}$ emission along the viewing chords shown in figure 6 for the outer (left) and inner (right) divertor. The lower boxes show the extrapolated total radiated power using the inverse S/XB technique and the contributions by $\mathrm{D}^{0}$ and carbon. The spatial coordinate used is the separatrix distance of the hit point of the lines of sight at the target measured along the target, see figure 6. Experimental conditions and plasma parameters corresponding to figure 4. The line intensities are sampled with 1 ms exposure time and then coherent ELM averaged. The temporal evolution of the coherently averaged ELM is used to estimate the emission in the time interval from 0.2 till $0.8 \mathrm{~ms}$ with respect to the ELM start.

outer divertor, but not for the inner. For the inner divertor, the carbon atomic data are strongly $\mathrm{T}_{e}$ dependent for the low temperatures around $6 \mathrm{eV}$, and therefore the extrapolation to total radiated power fluxes is unreliable. To demonstrate this, the carbon radiated power is plotted also with 1.5 times the measured electron temperature used to evaluate the atomic data. The strong inferred carbon emission is systematically overestimated, since the ionisation time of $\mathrm{C}^{2+}$ is a few ms for these conditions and therefore longer than the ELM duration. The inferred deuterium radiation is much less temperature dependent in the $\mathrm{T}_{e}$ range typical during ELMs. It should be noted that the influx of $\mathrm{C}^{+}$is possibly underestimated by the method described above, but the effect on the inferred total radiation will be small since $\mathrm{C}^{+}$radiates much less than $\mathrm{C}^{2+}$ for these divertor conditions. For both divertors, the deuterium radiation dominates during the ELM, and therefore the spectroscopic extrapolation can be used to estimate the contribution of the radiated power to the thermographic power flux.

Since a deconvolution of the radiated power profile is not possible with this set-up of lines of sight, a simple estimate is used to calculate the radiative power deposition on the target plates: one half of the power per $\mathrm{m}^{2}$ emitted along the line of sight is assumed to be deposited on the divertor target. This estimate is good if the major part of the radiation is close to the target, which is an acceptable approximation. For the conditions of figure 7 , the radiation energy on the lower targets amounts to $0.3 \mathrm{~kJ}$ for the outer and $0.9 \mathrm{~kJ}$ for the inner lower divertor for the time interval of $\mathrm{dt}=0.2-0.8 \mathrm{~ms}$ (summarized values are given below in table 1). Due to the caveats mentioned above (uncertainty in atomic data, marginal time resolution, spatial profiles), these numbers bear a considerable uncertainty (factor 2-3). Despite this uncertainty margin, it is clear that radiation does not dominate the power load during the ELM. In the far inner SOL and PFR regions, the inferred radiation is expected to be the dominant contribution to the power load [27]. 


\subsection{Electric current and sheath potential}

The standard power calculation from Langmuir probes does not take into account net electric currents flowing in and out of the targets, causing an imbalance of electron and ion fluxes. The net target current integrated over the lower divertor tile is measured by shunts [37] and shown in figure 8. The spatial extent of the measurement is up to ds $\mathrm{s}_{s e p} \approx 0.15 \mathrm{~m}$ and corresponds to the region where Langmuir probe and thermography data are available and integrated to obtain total ELM energies and ion fluxes. Due to a low-pass filter in the amplifier electronics, the time resolution of the electric current signal, which was sampled with $5 \mathrm{kHz}$ digitization rate, was limited to $1 \mathrm{~ms}$. A recent upgrade of the diagnostic to full $100 \mathrm{kHz}$ time resolution revealed a very similar duration of the SOL current for comparable ELMs, showing that the quite long duration of the current pulse shown in figure 8 was not caused by the electronics. The electric current flowing during the ELM is significant and can approach the ion saturation current. The fluctuation of the current signal around its smoothed value is a consequence of the coherent mapping and displays the variation between the 166 overlaid individual ELMs. The current balance between inner and outer divertor is not perfect. Possible reasons for the deviation are toroidal asymmetries or the loss of current into plasma facing componenents not equipped with shunt measurements [38].

Allowing for non-ambipolar, net electric fluxes and neglecting secondary electron emission the power derived from the LP can be written as

$q=\Gamma_{i} \cdot\left(2 k T_{i}+e V_{\text {sh }}+E_{\text {rec }}\right)+\Gamma_{e} \cdot 2 k T_{e}$

The electron flux density, $\Gamma_{e}$ is determined from the ion saturation current and the electric current. The main unknown quantities in this equation are the ion temperature $\mathrm{T}_{i}$ and the sheath potential $\mathrm{V}_{s h} . \mathrm{E}_{\text {rec }}$ is the recombination energy at the target surface. For standard sheath theory without current flowing, the plasma sheath potential $V_{s h}$ is determined by fast Maxwell tail electrons which negatively charge the plasma facing surface. To preserve ambipolarity, the negative surface charge repels most of the electrons close to the plate, and a positive space charge results. With the target as zero potential reference, a positive potential at the sheath entrance results, $V_{s h}=3 \mathrm{kT}_{e} / \mathrm{e}$ [26]. With a current flowing, the sheath potential is expected to deviate from its standard value by $\Delta \mathrm{V}_{s h}=\mathrm{V}_{s h}-3 \mathrm{kT}_{e} / \mathrm{e}$.
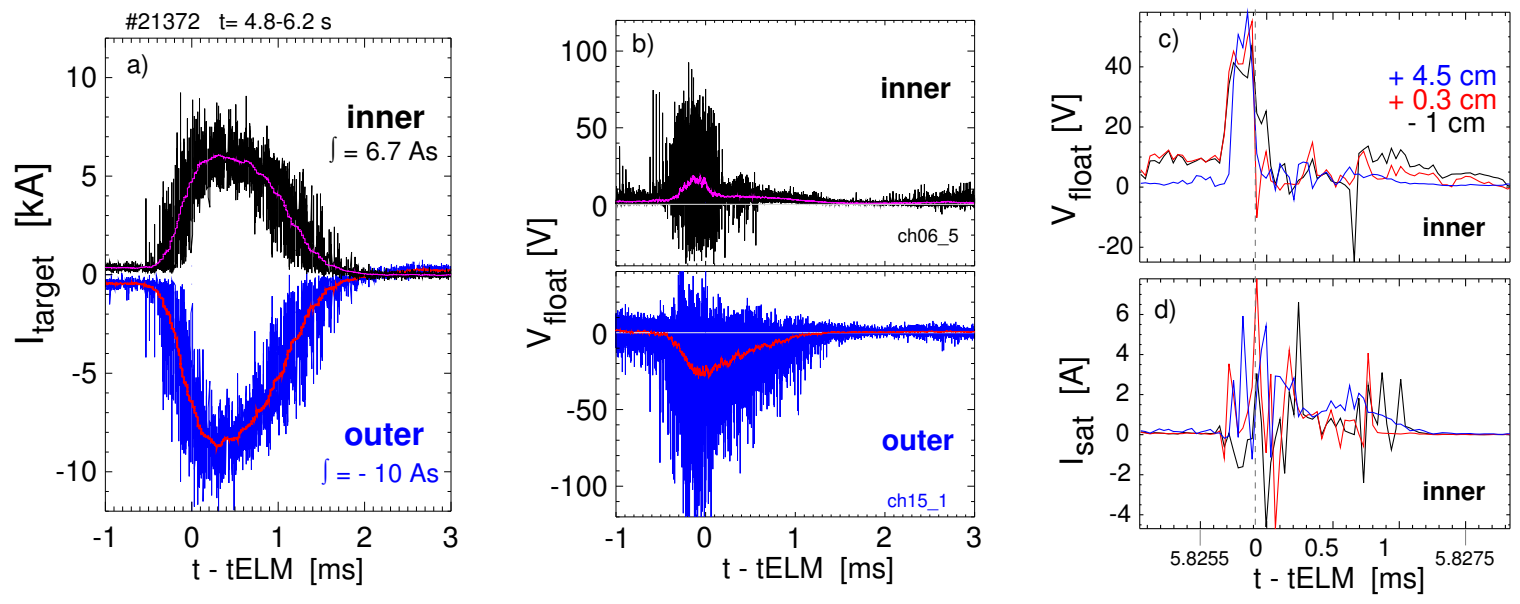

Figure 8. Coherent ELM averaged a) electric current into inner and outer divertor from tile shunt measurements and b) floating potential measured at the divertor SOL closely outside the separatrix c) and d) show floating potential and ion saturation current for a typical individual ELM at 3 positions in the inner divertor. The dashed line indicates the ELM $t=0$ time as derived from the thermography signal. The time resolution for floating potential and ion saturation current is $35 \mu$ s, the size of the probe electrode is $5 \mathrm{~mm}$ in poloidal and $25 \mathrm{~mm}$ in toroidal direction. A current of $1 \mathrm{~A}$ corresponds to an ion flux of $5 \cdot 10^{22} \mathrm{~m}^{-2} \mathrm{~s}^{-1}$. 
To demonstrate the time behaviour of a typical individual ELM, figure 8 also shows floating potential and ion saturation current for different locations at the target. $\mathrm{V}_{f l}$ and $\mathrm{I}_{s a t}$ are strongly fluctuating during the ELM, even negative values of the ion saturation current appear. $\mathrm{j}_{\text {sat }}$ is measured between two probe tips separated by about $0.1 \mathrm{~m}$ in toroidal direction with a bias voltage of $72 \mathrm{~V}$. The negative values of $\mathrm{I}_{\text {sat }}$ suggest a dynamic plasma potential variation in excess of this voltage over a corresponding distance. The strongly fluctuating nature of the ion saturation current, electric current and floating potential is in particular pronounced for low density conditions. The ratio of the electric current and the ion saturation current, $\mathrm{I}_{0} / \mathrm{I}_{\text {sat }}$, measured with Langmuir probes operated at the same poloidal locations as triple probe and unbiased at target potential, fluctuates around the value 1 at low density. Reduced fluctuations and values close to zero are observed at high density.

\section{Quantitative analysis of the ELM power in-out asymmetry}

The presence of the large net ELM electric current suggests two possible contributions to the power in-out asymmetry. First, directed pedestal ion losses towards the inner divertor could provide the positive charge flow and additional power deposition. The negative radial electric field in the edge transport barrier (ETB) is supposed to cause the direction of the lost ion orbits towards the inner target [10] [12].

As second contributor, a loop voltage is expected to show up along the field lines close to the separatrix during the ELM as described below. When the ELM starts, temperature and density gradients in the pedestal region flatten considerably. As a consequence, the driving term for the pedestal bootstrap current [39], which can be as large as $10 \%$ of the plasma current, is considerably reduced. According to Lenz' rule a toroidal loop voltage develops simultaneously which drives the current necessary to conserve the poloidal magnetic flux in the edge plasma. The loop voltage can immediately reach the region of open field lines. The normal current diffusion time for separatrix parameters is about $0.2 \mathrm{~ms}$ for a distance of $1 \mathrm{~cm}$, but a faster redistribution of the current is possible to take place during the nonlinear evolution of the ELM instability [40]. The divertor ends of the field line act like a double Langmuir probe, and the voltage drop mainly occurs at the ion end, which is the inner divertor for standard field direction. This voltage can drive an electric current and accelerate the ions towards the inner target, resulting in an increased ion power load. $\mathrm{V}_{s h}$ can become much larger than $3 \mathrm{kT}_{e} / \mathrm{e}$ at the ion end (inner divertor) if the electric current approaches the ion flux, since the ion flux is limited and the electrons are almost completely repelled. No pronounced variations of the sheath potential are expected at the electron end, since the high electron mobility allows considerable increase of the electron current. The voltage associated with the ETB profile flattening has the right sign to explain the ELM power asymmetry for the various combinations of field directions.

To learn more about the relative contribution of the mechanisms described above on the ELM power in-out asymmetry, a quantitative evaluation of the different power channels is required. For this purpose, various quantities of coherent averaged ELMs like that shown in figure 4 have been integrated/averaged from 0.2-0.8 ms with respect to the ELM start time for different discharges and flattop phases. It is well known that at least a fraction of the ELM power is released in filamentary structures [41], which can be observed in the SOL profiles of the IR power load measurements [42]. The nonaxisymmetric stripes observed in the heat flux measurement are smeared out to a toroidally symmetric power deposition pattern on the target at the strike lines [9]. Due to the coherent time averaging over a large number of ELMs, the data shown here average out possible effects of filaments.

The experimental data are summarized in table 1. The loss power from the product of ELM energy $\triangle \mathrm{E}_{E L M}$ and ELM frequency $\mathrm{f}_{E L M}$ corresponds to about $20 \%$ of the total heating power as typical in ASDEX Upgrade [43]. The ion temperature $\mathrm{T}_{i}$ and the sheath potential $\mathrm{V}_{s h}$ are for the time being regarded unknown quantities. Integrating equation 2 over time

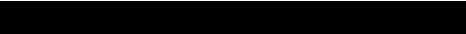




\begin{tabular}{|c|c|c|c|c|c|c|c|c|c|c|c|}
\hline $\begin{array}{l}\text { shot } \\
\text { time }\end{array}$ & $\begin{array}{l}\triangle \mathrm{E}_{E L M} \\
(\mathrm{MHD}) \\
\end{array}$ & $\mathrm{f}_{E L M}$ & $\begin{array}{c}\mathrm{dN} \\
(\mathrm{IDA})\end{array}$ & div & $\begin{array}{c}\mathrm{E}_{E L M} \\
(\mathrm{IR}) \\
\end{array}$ & $\begin{array}{l}\mathrm{E}_{\text {rad }} \\
\text { (est.) }\end{array}$ & $\mathrm{N}_{i o n}$ & $\mathrm{~N}_{\text {charge }}$ & $\mathrm{T}_{e}$ & $\begin{array}{c}\Delta \mathrm{V}_{s h} \\
\left(\mathrm{~T}_{i}=\mathrm{T}_{e}\right)\end{array}$ & $\begin{array}{c}\mathrm{T}_{i} \\
\left(\mathrm{~V}_{s h}=3 \mathrm{kT}_{e} / \mathrm{e}\right)\end{array}$ \\
\hline & {$[\mathrm{kJ}]$} & {$[\mathrm{Hz}]$} & [As] & & {$[\mathrm{kJ}]$} & {$[\mathrm{kJ}]$} & [As] & [As] & {$[\mathrm{eV}]$} & [V] & {$[\mathrm{eV}]$} \\
\hline 21372 & 15 & 120 & 6.6 & in & 3.9 & 0.9 & 8.3 & 4.4 & 4.8 & 319 & $164 \pm 80$ \\
\hline $4.8-6.2 \mathrm{~s}$ & $"$ & " & $"$ & out & 2.5 & 0.3 & 13.7 & -7.0 & 20.3 & -16 & $12 \pm 50$ \\
\hline 21372 & 13 & 81 & 7.2 & in & 6.1 & 0.7 & 10.0 & 4.8 & 6.0 & 490 & $251 \pm 100$ \\
\hline $2.8-4.0 \mathrm{~s}$ & $"$ & $"$ & $"$ & out & 2.3 & 0.2 & 11.9 & -8.0 & 21.7 & -19 & $12 \pm 50$ \\
\hline 21333 & 13 & 102 & 6.7 & in & 4.3 & 0.9 & 8.2 & 4.1 & 4.9 & 371 & $191 \pm 90$ \\
\hline $4.8-6.2 \mathrm{~s}$ & $"$ & $"$ & $"$ & out & 2.1 & 0.3 & 14.5 & -6.5 & 19.8 & -46 & $-3 \pm 40$ \\
\hline 21333 & 15 & 76 & 8.0 & in & 6.2 & 0.9 & 10.1 & 4.8 & 5.8 & 476 & $244 \pm 100$ \\
\hline $2.6-4.1 \mathrm{~s}$ & $"$ & $"$ & $"$ & out & 2.1 & 0.2 & 12.2 & -7.3 & 21.0 & -30 & $6 \pm 50$ \\
\hline 21301 & 5.5 & 146 & 3.8 & in & 2.3 & 0.7 & 6.4 & 2.7 & 4.6 & 208 & $108 \pm 60$ \\
\hline $5.2-6.4 \mathrm{~s}$ & $"$ & $"$ & $"$ & out & 1.1 & 0.3 & 15.3 & -4.8 & 14.9 & -75 & $-23 \pm 30$ \\
\hline
\end{tabular}

Table 1. ELM global and divertor parameters for discharges with varying heating power (5 - $10 \mathrm{MW})$ and gas puff. $\Delta \mathrm{E}_{E L M}$ is the total ELM energy loss from equilibrium analysis. $\mathrm{dN}$ is the total number of electrons/ions lost during the ELM inside the separatrix, see figure 10. All other data are time-integrated or time averaged from $\mathrm{dt}=0.2-0.8 \mathrm{~ms}$ with respect to the ELM start time and spatially integrated or averaged from the separatrix into the SOL by about $0.15 \mathrm{~m}$ along the target. The energy deposition due to radiation, $\mathrm{E}_{\text {rad }}$, which contributes to the power deposition measured by IR thermography, $\mathrm{E}_{E L M}$, is estimated from spectroscopic measurements as described in section 4.1. In the last two columns, the deviation from standard sheath potential, $\Delta \mathrm{V}_{s h}$, is calculated for the assumption $\mathrm{T}_{i}=\mathrm{T}_{e}$ and the ion temperature $\mathrm{T}_{i}$ is calculated for standard $\mathrm{V}_{s h}=3 \mathrm{kT}_{e} / \mathrm{e}$ according to eq. 3 . The uncertainty for $\Delta \mathrm{V}_{s h}$ in $\mathrm{V}$ is $1.5-2$ times the uncertainty given for $\mathrm{T}_{i}$ in $\mathrm{eV}$ in the last column. $\mathrm{N}_{\text {ion }}=\int d t d s 2 \pi R j_{\text {sat }}, \mathrm{N}_{\text {charge }}=\int d t I_{\text {target }}$. $\mathrm{B}_{t}=-2 \mathrm{~T}, \mathrm{q}_{95}=3.3$.

(0.2-0.8 ms) and space (well diagnosed target region), the target power balance can be closed by choosing the required ion temperature $\mathrm{T}_{i}$ or deviation $\Delta \mathrm{V}_{s h}$ of the sheath potential from $3 \mathrm{kT}_{e} / \mathrm{e}$ :

$2 T_{i}+\Delta V_{\text {sh }}=\left(E_{E L M}-E_{\text {rad }}\right) / N_{\text {ion }}-5 T_{e}+2 T_{e} N_{\text {charge }} / N_{\text {ion }}-E_{\text {rec }}$

$\mathrm{T}_{i}, \mathrm{~T}_{e}$ and $\mathrm{E}_{\text {rec }}$ are expressed here in electron volts $(\mathrm{eV})$ and $\mathrm{N}_{\text {ion }}$ and $\mathrm{N}_{\text {charge }}$ in As to comply with table 1 . A value of 14 $\mathrm{eV}$ is assumed for the ion recombination energy at the target, $\mathrm{E}_{r e c}$. Fixing either $\mathrm{T}_{i}$ or $\Delta \mathrm{V}_{s h}$ one of these parameters allows to calculate the other one from the experimental data. Accordingly, the last two columns of table 1 show the required additional sheath potential drop $\Delta \mathrm{V}_{s h}$ assuming $\mathrm{T}_{i}=\mathrm{T}_{e}$ and the ion temperature $\mathrm{T}_{i}$ assuming $\Delta \mathrm{V}_{s h}=0$ in order to satisfy equation 3 .

The additional sheath potential drop $\Delta \mathrm{V}_{s h}$ in the inner divertor required to explain the power asymmetry is considerably larger than the measured $\mathrm{V}_{f l}$ as shown in figure 8 , which is taken as a measure for the deviation of $V_{s h}$ from $3 \mathrm{kT} /$ e. As shown in figure 9, the inferred ion temperature to satisfy the power balance in the inner divertor is comparable to but smaller than the pedestal ion temperature (assuming $\mathrm{T}_{i} \approx \mathrm{T}_{e}$ ), making the preferential ion loss the dominating candidate. The negative values of $\mathrm{T}_{i}$ derived for some outer divertor cases lie within the uncertainties and are not expected to be real.

It should be noted also that a thermoelectric current during the ELM is expected to contribute to the net current through the targets. An estimation of the ELM thermoelectric current using measured SOL parameters and an analytical model [44] predicts thermoelectric currents of about $30 \%$ of the measured currents. The inferred values of $\mathrm{T}_{i}$ and $\Delta \mathrm{V}_{s h}$ of table 1 would only be slightly affected by the related variation of the sheath potential, since they are mainly determined by $\mathrm{E}_{E L M}$ and $\mathrm{T}_{e}$. 


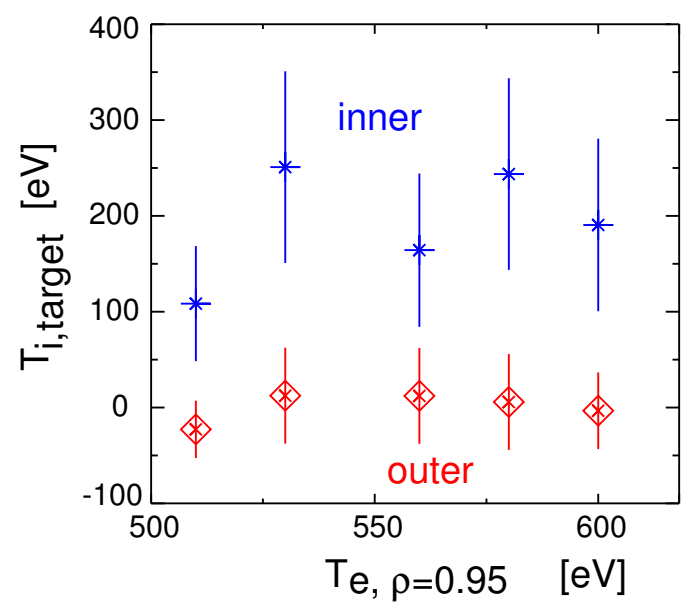

Figure 9. ELM ion temperatures at inner and outer target versus pre-ELM pedestal electron temperature at $\rho_{\text {pol }}=0.95$ obtained from a fit to edge Thomson scattering data. The ion temperatures are inferred from power/particle/current balance according to table 1 and equation 3.

The most critical quantity for the interpretation of the ELM power flow and the results presented in table 1 is the total deposited energy measured by the IR thermography system [5]. As discussed in section 4., in particular the derived total energy is expected to be a quite robust quantity. Integrating over an ELM duration of $2 \mathrm{~ms}$, the total deposited ELM energy measured by IR is also consistent with the total energy drop derived from equilibrium analysis, taking into account the reheating energy dt· $\left(\mathrm{P}_{\text {heat }}-\mathrm{P}_{\text {rad }}\right)$ during the integration time and a main chamber ELM power deposition of the order of 25 $\%$ of the ELM energy [45].

The low ion temperatures inferred in the outer divertor suggest the presence of a near-thermal plasma. Taking into acount the uncertainties of the individual measurments, the standard sheath model of equation 2 achieves satisfactory consistency. The presence of a net electric current out of the outer target (net electron flux into the target) suggests a reduction of the standard sheath potential drop by [20]

$\Delta V_{\text {sh }} \approx k T_{e} / e \ln \left(1+N_{\text {charge }} / N_{\text {ion }}\right)$

The reduced sheath potential drop at the outer target would increase the inferred values of $\mathrm{T}_{i}$ shown in table 1 in the outer divertor by a few $\mathrm{eV}$. The increased sheath potential according to equation 4 evaluated at the inner target is small compared to the potential rise which would be required to explain the power load. This is still the case when the fluctuating nature of the current is taken into account, which can lead to large, short and localised peaks in $\Delta \mathrm{V}_{s h}$.

An interesting test, in combination with the ion loss model, is to compare the net positive charge collected at the inner target with the number of ions lost during the ELM from midplane profile measurements. Figure 10 shows electron density profiles obtained from coherent ELM averaging of data obtained by integrated data analysis (IDA) [46] of Li-beam and interferometer data with $0.2 \mathrm{~ms}$ temporal resolution. The erosion of the pedestal density profiles by the ELMs is well resolved, the separatrix serves as pivot point for the dynamic profiles. The high quality of the profile data allows to calculate the number of electrons lost during the ELM. In figure 11 this number is compared to the collected ion number at the inner target from $\mathrm{j}_{\text {sat }}$ and the collected net charges. It should be noted that we compare here the ELM 'flattop' values for the target data with the pre/after ELM density profiles. The total values over an ELM for the charges and currents are about a factor 1.5 larger than the shown values integrated over the time interval $\mathrm{dt}=0.2-0.8 \mathrm{~ms}$. The total ion flux at both targets, $\mathrm{N}_{i o n}$, is higher than the number of ions lost from the core, $\mathrm{N}_{\text {loss }}$, indicating high recycling conditions during the 


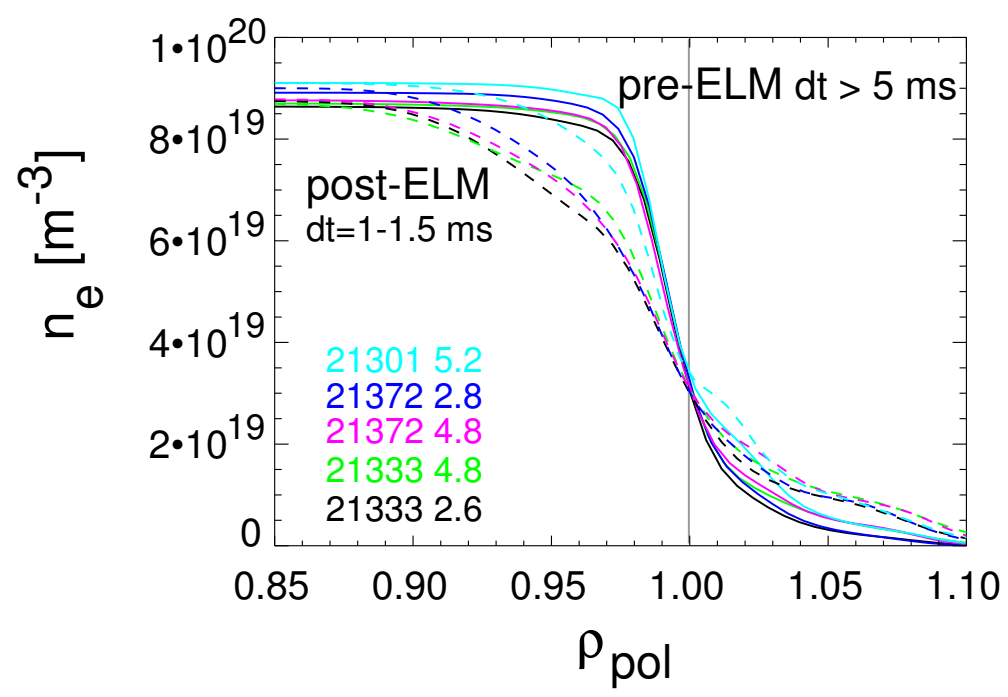

Figure 10. Electron density profiles in between ELMs (dt $>5 \mathrm{~ms})$ and right after the ELM (dt=1-1.5 ms) obtained from integrated data analysis of Li-beam and interferometer data. Labels indicate shot number and start time of the interval according to table 1.

ELM.

The charge collected at the inner target, $\mathrm{N}_{\text {charge }}$, coincides well with the absolute number of particles lost from the core. This finding supports the assumption that an important fraction of the ions lost in the pedestal region is directed towards the inner divertor. The ELMs characterised in table 1 are predominantly convective [22]: Constructing pre / past ELM edge pressure profiles using the density profiles shown in figure 10 and the pre-ELM electron temperature profiles (no ELM time resolution available for $\mathrm{T}_{e}$ ), the kinetic ELM energy drop for convective ELMs is obtained by volume integration. The resulting values lie between 67 and $84 \%$ of the stored energy drop from MHD analysis, indicating a large convective fraction of the ELM power.

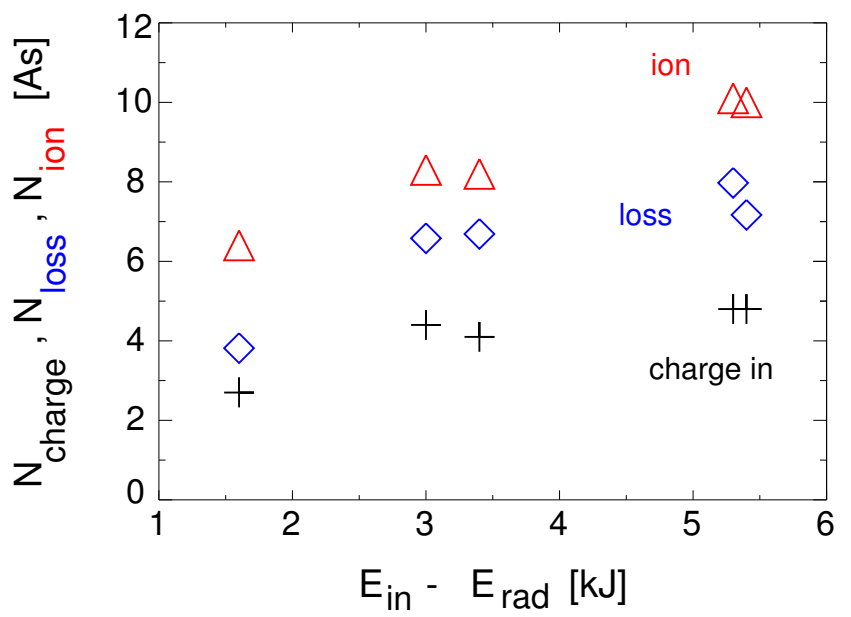

Figure 11. Ion and net charge numbers $\left(\mathrm{N}_{\text {ion }}, \mathrm{N}_{\text {charge }}\right)$ during ELMs $(\mathrm{dt}=0.2-0.8 \mathrm{~ms})$ and particles lost from the pedestal $\left(\mathrm{N}_{\text {loss }}\right)$ region just after the ELM (averaged dt=1-1.5 ms) versus the energy deposited at the inner target. An estimate for the contribution of radiation was subtracted from $\mathrm{E}_{\text {in }}$ according to table 1 . The electron $(\approx$ ion) loss from the pedestal region was obtained by volume integration of the profiles shown in figure 10 between $\rho_{p o l}=0.85$ and $\rho_{p o l}=1$. 


\section{Conclusions}

Divertor power fluxes derived from Langmuir probes and IR-thermography have been compared for different H-mode conditions in ASDEX Upgrade during and in between ELMs. In between ELMs, reasonable data consistency is obtained in the outer divertor for intermediate densities with the standard sheath model and taking into account a contribution of divertor radiation to the thermographic power flux. The power decay length in the outer divertor obtained from LP is shorter in comparison to IR, suggesting an effect of ions with $\mathrm{T}_{i}>\mathrm{T}_{e}$. The inner divertor strike zone is completely detached between ELMs, making data consistency checks difficult. During ELMs, the applicability of the probe theory used to evaluate the triple Langmuir probes is questionable. The ion saturation current exhibits a strongly fluctuation nature and adopts negative values transiently. This can be explained by transient floating potential differences between the two probe tips used for the $\mathrm{j}_{\text {sat }}$ measurement which exceed the biasing voltage. If these transient time phases are omitted from the data evaluation, (coherent) averaged LP data $\left(\mathrm{T}_{e}, \mathrm{n}_{e}, \mathrm{j}_{s a t}\right)$ can be derived during ELMs, albeit with reduced accuracy. However, the pronounced difference between Langmuir probe and thermographic power flux during ELMs in the inner divertor is too large to be attributed to diagnostic imperfections. The quantitative analysis of the ELM power load using various diagnostics suggests the directed loss of pedestal ions to the inner divertor as the most important contribution to the power in-out asymmetry. To explain the excess ELM power flux in the inner divertor by sheath acceleration, considerably higher sheath potentials would be required compared to the measured floating potentials during the ELM. The assumption of directed pedestal ion losses towards the inner divertor [10] [12] can explain the net charge flow measured by shunts for realistic values of the ion temperature. As a consequence, the net charge flow during type-I ELMs should not be dominated by the thermoelectric component under these conditions. Analysis of the ELM driven target current in the TCV tokamak suggested the right order of magnitude of the thermoelectric ELM current, with the ELMs being probably of type-III [17]. To really close the issue of the ELM divertor power load asymmetry, ion orbit following calculations [12] [47], that would be able to predict the ion velocity distribution at both targets for realistic ELM conditions, are required. Since such calculations should include the temporal evolution of the radial, poloidal and toroidal electric fields during an ELM, a self consistent coupling with a fluid code will probably be required.

\section{References}

[1] KALlENBACH, A. et al., Plasma Phys. Controlled Fusion 46 (2004) 431.

[2] LEONARD, A. et al., J. Nucl. Mater. 266-269 (1999) 109.

[3] CHANKIN, A. V. et al., Plasma Phys. Controlled Fusion 48 (2006) 839.

[4] FENSTERMACHER, M. et al., Plasma Phys. Controlled Fusion 45 (2003) 1597.

[5] HERRMANN, A. et al., J. Nucl. Mater. 313-316 (2003) 759.

[6] LEONARD, A. et al., J. Nucl. Mater. 313-316 (2003) 768.

[7] EICH, T. et al., Plasma Phys. Controlled Fusion 49 (2007) 573.

[8] LOARTE, A. et al., J. Nucl. Mater. 313-316 (2003) 962.

[9] EICH, T. et al., J. Nucl. Mater. 337-339 (2005) 669.

[10] FUNDAMENSKI, W. et al., Plasma Phys. Controlled Fusion 44 (2002) 761.

[11] FUNDAMENSKI, W. et al., J. Nucl. Mater. 337-339 (2005) 305.

[12] HAHN, S. et al., Phys. Plasmas 12 (2005) 102501.

[13] ROGNLIEN, T. and SHIMADA, M., J. Nucl. Mater. 313-316 (2003) 1000.

[14] STANGEBY, P. C., Nucl. Fusion 43 (2003) L1.

[15] ZHENG, L. et al., Phys. Rev. Lett. 100 (2008) 115001.

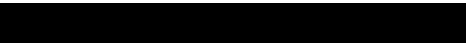


[16] TAKAHASHI, H. et al., Phys. Rev. Lett. 100 (2008) 205001.

[17] PITTS, R. A. et al., Nucl. Fusion 43 (2003) 1145.

[18] EICH, T. et al., J. Nucl. Mater. 363-365 (2007) 989.

[19] HARBOUR, P. et al., J. Nucl. Mater. 162-164 (1989) 236.

[20] STAEBLER, G. M. and HINTON, F. L., Nucl. Fusion 29 (1989) 1820.

[21] CHANKIN, A. V., J. Nucl. Mater. 241-243 (1997) 199.

[22] LOARTE, A. et al., Plasma Phys. Controlled Fusion 45 (2003) 1549.

[23] NEUHAUSER, J. et al., Nucl. Fusion 48 (2008) 045005.

[24] WEINLICH, M. and CARLSON, A., Phys. Plasmas 4 (1997) 2151.

[25] WISCHMEIER, M. et al., J. Nucl. Mater. 363-365 (2007) 448.

[26] STANGEBY, P., The Plasma Boundary of Magnetic Fusion Devices, Institute of Physics Publishing, Bristol and Philadelphia, 2000.

[27] FUCHS, J. et al., J. Nucl. Mater. 290-293 (2001) 525.

[28] KALLENBACH, A. et al., J. Nucl. Mater. 337-339 (2005) 381.

[29] HERRMANN, A. et al., J. Nucl. Mater. 313-316 (2003) 759.

[30] FUNDAMENSKI, W. et al., Nucl. Fusion 44 (2004) 20.

[31] NEUHAUSER, J. et al., Plasma Phys. Controlled Fusion 44 (2002) 855.

[32] KALLENBACH, A. et al., Divertor characterisation and data consistency in ASDEX upgrade, in Europhysics Conference Abstracts (Proc. of the 34th EPS Conference on Controlled Fusion and Plasma Physics, Warsaw, 2007), volume 31F, pages P-1.063, 2007.

[33] HERRMANN, A., J. Nucl. Mater. 337-339 (2005) 907.

[34] LOARTE, A. et al., Nucl. Fusion 47 (2007) S203.

[35] KALLENBACH, A. et al., Nucl. Fusion 39 (1999) 901.

[36] BEHRINGER, K. et al., Plasma Phys. Controlled Fusion 31 (1989) 2059.

[37] KALLENBACH, A. et al., J. Nucl. Mater. 290-293 (2001) 639.

[38] TAKAHASHI, H. et al., Nucl. Fusion 44 (2004) 1075.

[39] THOMAS, D. et al., Phys. Rev. Lett. 93 (2004) 065003.

[40] SOLANO, E. R. et al., J. Nucl. Mater. 337-339 (2005) 747.

[41] KIRK, A. et al., Plasma Phys. Controlled Fusion 49 (2007) 1259.

[42] EICH, T. et al., Plasma Phys. Controlled Fusion 47 (2005) 815.

[43] URANO, H. et al., Plasma Phys. Controlled Fusion 45 (2003) 1571.

[44] KALLENBACH, A. et al., J. Nucl. Mater. 290-293 (2001) 639.

[45] HERrmanN, A. et al., Plasma Phys. Controlled Fusion 46 (2004) 971.

[46] FISCHER, R. and DINKLAGE, A., Rev. Sci. Instrum. 75 (2004) 4237.

[47] HYNÖNEN, V. et al., Plasma Phys. Controlled Fusion 49 (2007) 151. 